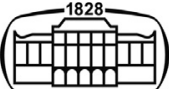

AKADÉMIAI KIADÓ

Acta Microbiologica et Immunologica Hungarica

68 (2021) 1, 1-6

DOl:

$10.1556 / 030.2021 .01366$

(c) 2021 The Author(s)

\section{ORIGINAL RESEARCH}

PAPER

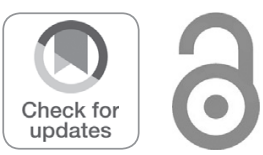

\title{
Interactions between immune system and the microbiome of skin, blood and gut in pathogenesis of rosacea
}

\author{
MARIE ISOLDE JOURA ${ }^{1}$, ALEXANDRA BRUNNER ${ }^{1}$, \\ ÉVA NEMES-NIKODÉM ${ }^{2}$, MIKLÓS SÁRDY ${ }^{1}$ and \\ ESZTER OSTORHÁZI ${ }^{1,3 *}$ (๑) \\ ${ }^{1}$ Department of Dermatology, Venereology and Dermatooncology, Semmelweis University, \\ Budapest, Hungary \\ ${ }^{2}$ Department of Biochemistry, Semmelweis University, Budapest, Hungary \\ ${ }^{3}$ Department of Medical Microbiology, Semmelweis University, Budapest, Hungary
}

Received: December 10, 2020 • Accepted: December 30, 2020

Published online: January 29, 2021

\begin{abstract}
The increasingly wide use of next-generation sequencing technologies has revolutionised our knowledge of microbial environments associated with human skin, gastrointestinal tract and blood. The collective set of microorganisms influences metabolic processes, affects immune responses, and so directly or indirectly modulates disease. Rosacea is a skin condition of abnormal inflammation and vascular dysfunction, and its progression is affected by Demodex mites on the skin surface. When looking into the effects influencing development of rosacea, it is not only the skin microbiome change that needs to be considered. Changes in the intestinal microbiome and their circulating metabolites, as well as changes in the blood microbiome also affect the progression of rosacea. Recent research has confirmed the increased presence of bacterial genera like Acidaminococcus and Megasphera in the intestinal microbiome and Rheinheimera and Sphingobium in the blood microbiome of rosacea patients. In this review we discuss our current knowledge of the interactions between the immune system and the skin, gut and blood microbiome, with particular attention to rosacea diagnostic opportunities.
\end{abstract}

\section{KEYWORDS}

blood microbiome, gut microbiome, immune system, rosacea, skin microbiome

\section{INTRODUCTION}

Rosacea is a chronic inflammatory skin disease that primarily affects women over 30 years of age [1]. The prevalence of rosacea is around $5 \%$ in the adult population worldwide [2].The disease progresses in episodes. Diagnostic criteria of rosacea include primary features, such as transient or persistent facial erythema, teleangiectasias, papules and pustules (usually in centrofacial distribution) and secondary features, such as a stinging or burning of the skin, oedema, tarsal conjunctivitis, ocular dryness and rhinophyma. Rosacea can be classified into four basic stages: erythemato-teleangiectatic, papulopustular, phymatous, and ocular rosacea, with the less prevalent form of granulomatosus rosacea [3]. Possible pathological mechanisms responsible for rosacea include dysregulation of the innate and possibly the acquired immune system [4], disrupted neurovascular signal transmission [5], degeneration of connective tissue elements, nutritional and chemical factors, UV and sun exposure, functional disorders of the pilosebaceous unit and infective microbes [6].

The highly sensitive and complex interaction between the immune conditions and the intestinal, blood or cutaneous microbes possibly influences the progression and therapeutic options of rosacea. 


\section{Dysregulation of innate and acquired immune system}

The intact skin surface as a physical barrier is important for the integrity of the innate immune system. The sensitive skin of rosacea patients results in decreased tolerance to commonly used topical agents. The increase of transepidermal water loss, or the directly caused breaches by Demodex mites lead to disturbances in the epidermal barrier which represent the major contributing factor in the pathogenesis of rosacea [7].

The cells of the innate immune system in the skin express pattern recognition receptors (PRRs) that recognize damage-associated or pathogen-associated molecular patterns. Among the PRRs, toll-like receptor 2 (TLR-2) expression is highly increased in rosacea patients in contrast to healthy controls or psoriatic patients [8]. TLR-2 is activated by external stimuli such as the chitin of Demodex mites or bacterial products. Activation of TLR-2 triggers keratinocytes to produce proinflammatory cytokines and chemokines including IL-8, IL- $1 \beta$, and TNF- $\alpha$. Elevated levels of cytokines and chemokines lead to chemotaxis of neutrophils into the skin and vascular hyper-reactivity [9]. Additionally, TLR-2 is known to increase the expression of serine protease KLK-5.

Cathelicidin is an antimicrobial protein (AMP) that can be found in neutrophils, mast cells, macrophages and monocytes and in lamellar bodies of keratinocytes [10]. KLK-5 serine protease together with proteinase-3 are responsible for cleaving inactive cathelicidin into the active peptide form LL-37. In contrast to healthy people, rosacea patients have an increased baseline expression of cathelicidin and KLK-5 [11, 12] LL-37 works against bacteria, fungi and parasites. In addition to its antimicrobial activity, LL-37 modulates neutrophil chemotaxis, and stimulates cytokine and chemokine release from mast cells [13]. The degranulation of mast cells leads to the exhibition of clinical features of rosacea, such as telangiectasia, erythema, and inflammation [14]. Moreover, LL-37 affects angiogenesis through enhancing the proliferation of endothelial cells and promotes expression of extracellular matrix components [15].

The adaptive immunity likewise exhibits dysregulated functions in rosacea patients. Inflammatory infiltration in rosacea leads to an increase in the amounts of Th1 and Th17 cells. The cytokine production of Th17 induces angiogenesis and also affects the elevated expression of LL-37 [4].

Approximately $10-20 \%$ of inflammatory cell infiltration in rosacea is composed of $\mathrm{CD} 20+\mathrm{B}$ cells [16]. B cells through the production of fibrogenic cytokines such as IL-6 and TGF- $\beta$ via TLR can stimulate fibrotic changes in the skin as it was shown in phymatous rosacea [17] (Fig. 1).

\section{The role of microbes in the pathogenesis of rosacea}

Novel microbiological diagnostic methods unearthed the association between certain microbes and rosacea disease. The simplest microscopic method, methylene blue stained adhesive tape preparation from rosacea skin surface clearly shows the accumulated amount of Demodex mites (Fig. 2.).
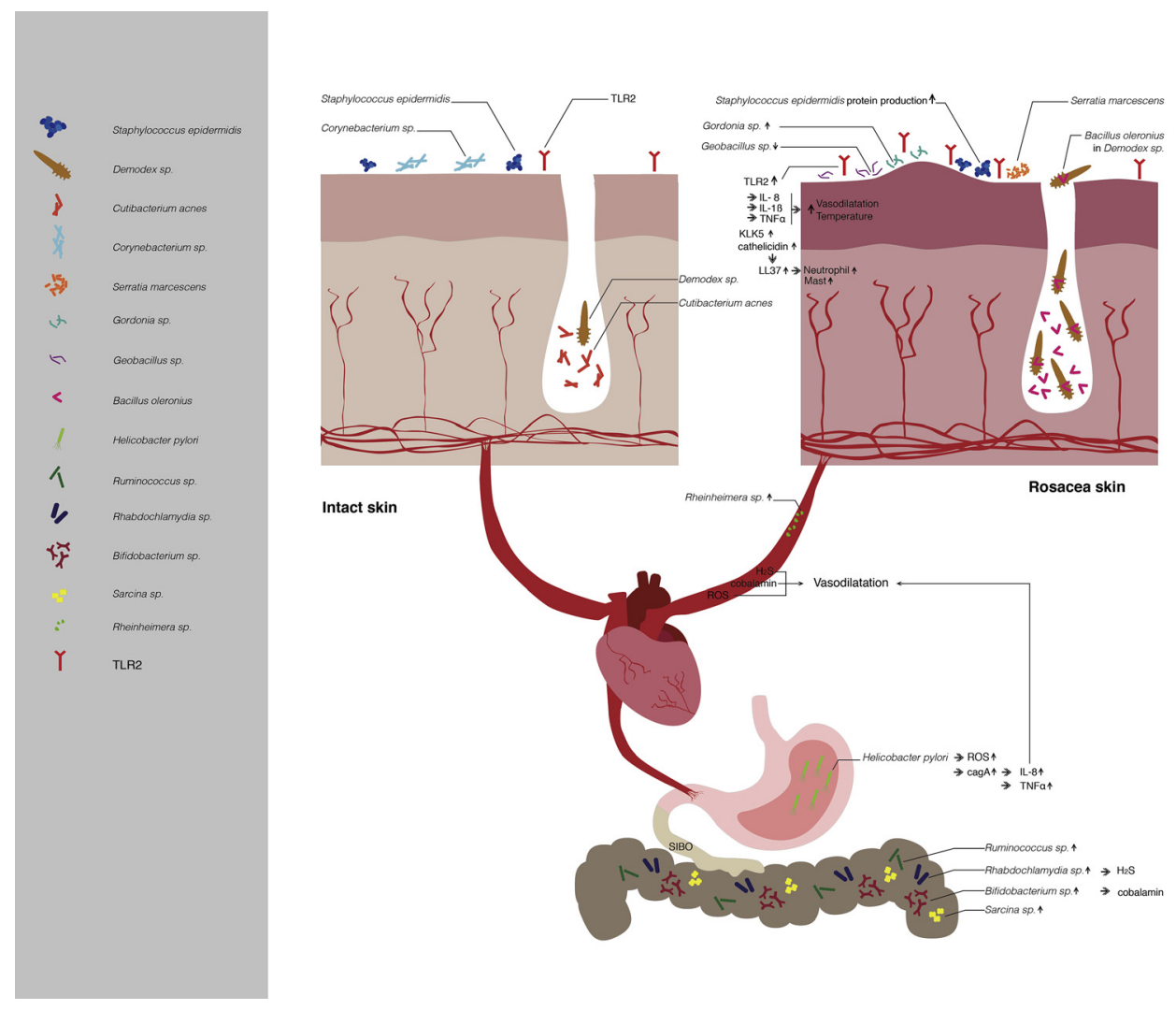

Fig. 1. Interaction between microorganisms and immunreactions in rosacea affected skin 


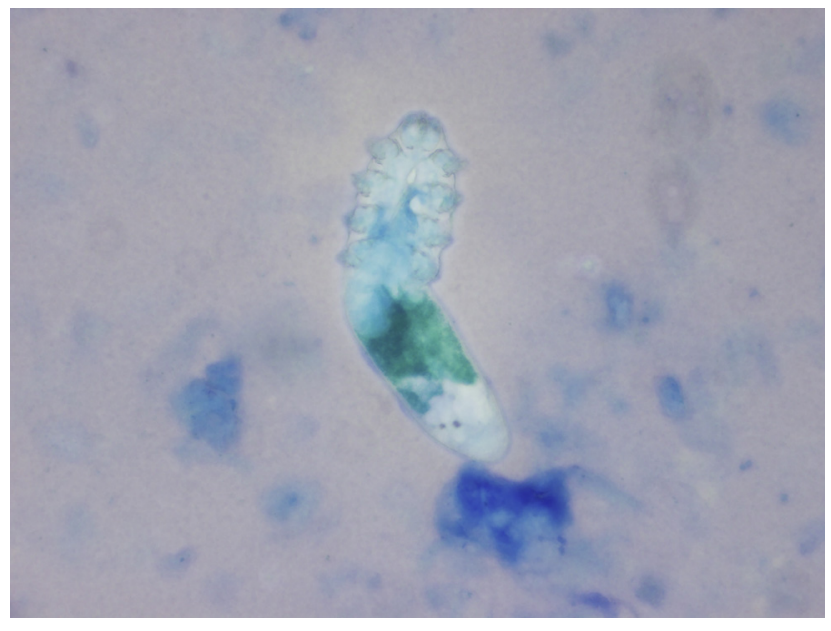

Fig. 2. Microscopical picture of Demodex folliculorum, methilenblue staining

The correlation between the presence of mites and rosacea were described in 1932 [18].

Using traditional aerobe culture methods, Staphylococcus epidermidis was frequently isolated from folliculi of biopsy samples of rosacea patients. As S. epidermidis is one of the most common bacterial species of the normal skin flora, its pathogenic role in rosacea is questionable. Because the facial skin is warmer in rosacea patients than in healthy individuals and S. epidermidis strains cultured at higher temperature secrete different proteins in different quantities, the pathogenic role of these proteins can likely be confirmed [19].

A passive transfer of additional micro-organisms from Demodex folliculorum was speculated after Bacillus oleronius, a Gram-negative rod-shaped bacterium had been successfully isolated from the mite obtained from a papulopustular rosacea patient [20]. Patients with erythematoteleangiectatic rosacea show serum reactivity to the 62 - and $83-\mathrm{kDa}$ proteins of $B$. oleronius, suggesting that these highly immunogenic proteins may play a role in the induction of this condition [21]. Exposure of neutrophils to proteins from B. oleronius resulted in degranulation and the production of inflammatory cytokines $[22,23]$. The increased temperature of the inflamed skin of rosacea patients alters the growth and increases the 62-kDa protein production of B. oleronius [24] and thus, continuously re-stimulates the inflammatory response.

Helicobacter pylori infection was suggested to play a role in the aethiopathogenesis of rosacea. However, the relationship is debated arguments ranging from coincidental, plausible or undecided, depending upon the examination method and the patient population in which the presence of the bacterium was tested. Using C [14]-urea-breath test as diagnostic tool the colonization rate of $H$. pylori was significantly higher in rosacea patients than in the control group $[25,26]$. Several reports showed seropositivity to H. pylori in rosacea individuals [27-29], although this claim is not universally accepted [30-32]. H. pylori produces ROS (reactive oxygen species) including NO (nitric oxide), and rosacea patients showed higher ROS in plasma than controls $[33,34]$. NO is important in a number of different physiological processes in the skin, including vasodilatation, inflammation, and immune modulation. It was postulated that $\mathrm{NO}$ produced by $\mathrm{H}$. pylori might cause the flushing and erythema associated with rosacea, or that it may have a pathogenic role in the inflammation seen in rosacea [35]. Furthermore, H. pylori can express cagA cytotoxin which induces express of TNF- $\alpha$ and IL-8, and subsequently a series of inflammatory reactions [36].

\section{New perspectives with New Generation Sequencing}

DNA sequencing studies have expanded our insights and understanding of the ecological and functional characteristics of the microbiome associated with different sites of the human body. It is estimated that humans harbour approximately 100 trillion microorganisms belonging to various domains, including viruses, bacteria, archaea, fungi, and protozoans [37]. Most of our knowledge of microbial communities in areas of the human body was obtained through non-culture-based testing of bacterial DNA. The bacterial $16 \mathrm{~S}$ rRNA gene region consists of hypervariable and conserved loci allowing the construction of universal primers for bacterial detection and identification [38]. In addition to traditional detection methods, this new generation sequencing method has highlighted the importance of some bacterial genera from the skin, gut and blood microbes in the pathogenesis of rosacea.

\section{Relationship between skin microbiome composition and rosacea}

The three different skin microenvironments - dry, moist and sebaceous - and their associated microbiomes have a characteristic topography in each individual [39]. In these three skin microenvironments three bacterial phyla - Firmicutes, Actinobacteria and Proteobacteria - represent more than $90 \%$ of all the resident taxa [40]. The bacterial species alpha diversity is widest in dry and narrowest in sebaceous skin environments [41]. In healthy individuals Staphylococcus and Corynebacterium genera preferring high humidity occur more frequently in moist areas, whereas lipophilic Propionibacterium genus tends to colonize sebaceous areas [42].

Skin disorders may be influenced by microbiome associated with different microenvironments. Symptoms of acne and rosacea occur in sebaceous areas, body odour and atopic dermatitis in moist areas and psoriasis in dry microenvironments [39].

While acne and rosacea may display similar clinical characteristics in sebaceous areas, the clinical courses of each condition are quite distinct. Suspected by Thompson and co-workers, differences in the skin microbiota may be related to different pathophysiological courses of acne and rosacea. In the case of microbiome analysis the distribution of bacterial taxa showed that Proteobacteria was the most abundant phylum in acne patients, whereas Actinobacteria was the most abundant phylum in rosacea patients. 
According to species-level comparison Serratia marcescens and Cutibacterium acnes (formerly Propionibacterium acnes) were each present in significantly higher mean relative abundance in rosacea patients compared to acne patients [43].

In erythemato-telangiectatic rosacea and papulopustular rosacea increased density of Demodex mites on the skin has long been observed. This mite utilizes sebum as a food source and other nutritional sources may be cellular debris or bacteria such as Cutibacterium acnes that reside in the pilosebaceous unit [39]. Demodex mites and the associated Bacillus oleronius bacteria trigger inflammatory pathways in rosacea patients [23].

Zaidi and co-workers tested the correlation between bacterial species relative percentage abundance (RPA) and the severity of rosacea using the score established by the National Rosacea Society (NRS). A significant $(P<0.05)$ correlation was found between NRS score and the RPA of 9 genera: Gordonia, Janibacter, Jeotgalicoccus, Tepidimonas, Dietzia, Wautersiella, Mycobacterium, Geobacillus and Weissella. Multivariate random effect Poisson regression (REPR) analyses demonstrated that a $10 \%$ increase in RPA of Gordonia or a $10 \%$ decrease in RPA of Geobacillus significantly increase the NRS score [44].

Rainer and co-workers compared the skin microbiome of rosacea-affected skin and healthy skin in a study based on 19 rosacea patients. Campylobacter ureolyticus, Cornyebacterium kroppenstedtii and Prevotella intermedia were found more common in patients with a mixed papulopustular-erythema-allergic form of rosacea [45].

\section{Relationship between gut microbiome composition and rosacea}

A link between rosacea and gastrointestinal diseases - such as gastroesophageal reflux, irritable bowel syndrome or small bowel bacterial overgrowth (SIBO) -have been demonstrated mainly by population based studies [46-49]. In a prospective study with a three-year follow-up of Agnoletti et al., SIBO significantly prevailed in papulopustular rosacea, while H. pylori infection in erythrosis [50]. The association is also confirmed by studies that show an improvement in rosacea in connection with SIBO antibacterial therapy [51, 52].

Using the NGS method, only a few studies have so far examined the relationship between intestinal microbiome composition and rosacea. Chen and co-workers have observed significant reduction of faecal microbial richness in rosacea patients, with increased abundance of Rhabdochlamydia, Bifidobacterium, Sarcina, and Ruminococcus genera. In this study the discriminating reduced abundant genera were Lactobacillus, Megasphaerae, Acidaminococcus, Haemophilus, Roseburia and Clostridium [53]. In contrast the study of Nam et al. described an increased incidence of Lactobacillus, Megasphaerae and Acidaminococcus genera in rosacea patients [54]. The altered microbiome also influences the quantitative change of bacterial metabolic products. Chen and co-workers speculated that faecal microbial composition might be related to sulfur metabolism, cobalamin transport, and carbohydrate transport [53]. The elevated hydrogene sulfide $\left(\mathrm{H}_{2} \mathrm{~S}\right)$ concentration in blood might shift the thiol/thiol disulfide balance toward thiol disulfide excess, resulting in a less effective protection against oxidative stress in rosacea patients. Sener and co-workers confirmed that the mean disulfide/native thiol ratio is significantly higher in rosacea patients than in controls [55]. The induction of rosacea symptoms is also known as an adverse side effect of B12 (cobalamin) therapy [56]. Not only overdosed therapy, but also altered intestinal microbiome can develop this detrimentally elevated cobalamin level.

\section{Relationship between blood microbiome composition and rosacea}

The circulation is a closed system and the blood in healthy organisms was first believed to be a sterile environment. Recent studies have detected microbial DNA in the blood of healthy humans, but it is still questioned whether they were derived from living or dead microbes or they simply contaminated the samples [57-59]. Additional studies have described significant differences in the blood microbiome of healthy individuals and individuals with various diseases including diabetes mellitus, schizophrenia, asthma or acute pancreatitis [60-63].

Only a limited number of studies of blood associated microbiome in dermatologic diseases like hidradenitis suppurativa [64], psoriasis [65] or rosacea [66] have been conducted. Faith's phylogenetic alpha diversity analysis showed a marginally significant difference between the blood microbiome of rosacea vs control patients. The relative abundance of Chromatiaceae and Fusobacteriaceae family was significantly elevated in the blood of rosacea patients. At the genus level 14 bacterial taxa had different abundance between the rosacea and healthy patients. The Rheinheimera genus differed increasingly across groups with significantly elevated abundance in rosacea patients [66].

Rheinheimera is a genus of Gram-negative aerobic bacteria. The occurrence of various species of the genus Rheinheimera has also been detected in marine and freshwater, industrial and urban wastewaters, plants and soil [67-71]. The exact pathophysiology of these bacteria in humans has not yet been fully clarified [72]. As Gram-negative bacteria, Rheinheimera contain lipopolysaccharides in their outer membrane, which may be involved in the pathogenesis of chronic inflammation in rosacea through the activation of TLR.

\section{CONCLUSION}

Rosacea is a chronic relapsing skin condition which can be controlled but does not have a traditional 'cure'. Among many other options (avoiding provocation factors, sun protection, vasoconstrictive topical care, azelaic acid, ivermectin, isotretinoin, laser or surgical measures), topical and oral antibiotic treatment are significant parts of anti-rosacea therapy. At this point, more information about the relationship between the triggering altered microbiome and the immune function involved in inflammation is needed. 
Accurate assessment of the protective and harmful microbiome composition is essential for targeted antibiotic or probiotic use in rosacea therapy.

Funding: No funding was received to assist with the preparation of this manuscript.

Conflicts of interest/Competing interests: All authors certify that they have no affiliations with or involvement in any organization or entity with any financial interest or nonfinancial interest in the subject matter or materials discussed in this manuscript.

\section{REFERENCES}

1. Thiboutot D, et al. Standard management options for rosacea: the 2019 update by the National Rosacea Society Expert Committee. J Am Acad Dermatol 2020; 82: 1501-10.

2. Gether L, Overgaard LK, Egeberg A, Thyssen JP. Incidence and prevalence of rosacea: a systematic review and meta-analysis. $\mathrm{Br} \mathrm{J}$ Dermatol 2018; 179: 282-9.

3. Gallo RL, et al. Standard classification and pathophysiology of rosacea: the 2017 update by the National Rosacea Society Expert Committee. J Am Acad Dermatol 2018; 78: 148-55.

4. Buhl T, et al. Molecular and morphological characterization of inflammatory infiltrate in rosacea reveals activation of Th1/Th17 pathways. J Invest Dermatol 2015; 135: 2198-208.

5. Schwab VD, et al. Neurovascular and neuroimmune aspects in the pathophysiology of rosacea. J Investig Dermatol Symp Proc 2011; 15: 53-62.

6. Yamasaki K, Gallo RL. The molecular pathology of rosacea. J Dermatol Sci 2009; 55: 77-81.

7. Woo YR, Lim JH, Cho DH, Park HJ. Rosacea: molecular mechanisms and management of a chronic cutaneous inflammatory condition. Int J Mol Sci 2016; 17.

8. Yamasaki $\mathrm{K}$, et al. TLR2 expression is increased in rosacea and stimulates enhanced serine protease production by keratinocytes. J Invest Dermatol 2011; 131: 688-97.

9. Gerber PA, Buhren BA, Steinhoff M, Homey B. Rosacea: the cytokine and chemokine network. J Investig Dermatol Symp Proc 2011; 15: 40-7.

10. Ahn CS, Huang WW. Rosacea pathogenesis. Dermatol Clin 2018; 36: $81-6$.

11. Niyonsaba F, Kiatsurayanon C, Chieosilapatham P, Ogawa H. Friends or Foes? Host defense (antimicrobial) peptides and proteins in human skin diseases. Exp Dermatol 2017; 26: 989-98.

12. Yamasaki $\mathrm{K}$, et al. Increased serine protease activity and cathelicidin promotes skin inflammation in rosacea. Nat Med 2007; 13: 975-80.

13. Yamasaki K, Gallo RL. Rosacea as a disease of cathelicidins and skin innate immunity. J Investig Dermatol Symp Proc 2011; 15: 12-5.

14. Muto Y, et al. Mast cells are key mediators of cathelicidin-initiated skin inflammation in rosacea. J Invest Dermatol 2014; 134: 2728-36.

15. Koczulla R, et al. An angiogenic role for the human peptide antibiotic LL-37/hCAP-18. J Clin Invest 2003; 111: 1665-72.

16. Cribier B. Rosacea under the microscope: characteristic histological findings. J Eur Acad Dermatol Venereol 2013; 27: 1336-43.

17. Pasare C, Medzhitov R. Control of B-cell responses by Toll-like receptors. Nature 2005; 438: 364-8.
18. Ayres S, Jr, Ayres S, 3rd. Demodectic eruptions (demodicidosis) in the human. 30 years' experience with 2 commonly unrecognized entities: pityriasis folliculorum (Demodex) and acne rosacea (Demodex type). Arch Dermatol 1961; 83: 816-27.

19. Dahl MV, Ross AJ, Schlievert PM. Temperature regulates bacterial protein production: possible role in rosacea. J Am Acad Dermatol 2004; 50: 266-72.

20. Lacey N, Delaney S, Kavanagh K, Powell FC. Mite-related bacterial antigens stimulate inflammatory cells in rosacea. Br J Dermatol 2007; 157: 474-81.

21. O’Reilly N, Menezes N, Kavanagh K. Positive correlation between serum immunoreactivity to Demodex-associated Bacillus proteins and erythematotelangiectatic rosacea. Br J Dermatol 2012; 167: 1032-6.

22. McMahon F, et al. Activation of neutrophils via IP3 pathway following exposure to demodex-associated bacterial proteins. Inflammation 2016; 39: 425-33.

23. O’Reilly N, Bergin D, Reeves EP, McElvaney NG, Kavanagh K. Demodex-associated bacterial proteins induce neutrophil activation. Br J Dermatol 2012; 166: 753-60.

24. Maher A, Staunton K, Kavanagh K. Analysis of the effect of temperature on protein abundance in Demodex-associated Bacillus oleronius. Pathog Dis 2018; 76.

25. Gravina A, et al. Helicobacter pylori infection but not small intestinal bacterial overgrowth may play a pathogenic role in rosacea. United Eur Gastroenterol J 2015; 3: 17-24.

26. Jorgensen AR, et al. Rosacea is associated with Helicobacter pylori: a systematic review and meta-analysis. J Eur Acad Dermatol Venereol 2017; 31: 2010-15.

27. Zandi S, Shamsadini S, Zahedi MJ, Hyatbaksh M. Helicobacter pylori and rosacea. East Mediterr Health J 2003; 9: 167-71.

28. Mini R, et al. Helicobacter pylori immunoproteomes in case reports of rosacea and chronic urticaria. Proteomics 2005; 5: 777-87.

29. Argenziano G, et al. Incidence of anti-Helicobacter pylori and antiCagA antibodies in rosacea patients. Int J Dermatol 2003; 42: 601-4.

30. Diaz C, O'Callaghan CJ, Khan A, Ilchyshyn A. Rosacea: a cutaneous marker of Helicobacter pylori infection? Results of a pilot study. Acta Derm Venereol 2003; 83: 282-6.

31. Szlachcic A. The link between Helicobacter pylori infection and rosacea. J Eur Acad Dermatol Venereol 2002; 16: 328-33.

32. Jones MP, Knable AL, Jr, White MJ, Durning SJ. Helicobacter pylori in rosacea: lack of an association. Arch Dermatol 1998; 134: 511.

33. Mashimo $M$, et al. Production of reactive oxygen species in peripheral blood is increased in individuals with Helicobacter pylori infection and decreased after its eradication. Helicobacter 2006; 11 : 266-71.

34. Baz K, et al. Plasma reactive oxygen species activity and antioxidant potential levels in rosacea patients: correlation with seropositivity to Helicobacter pylori. Int J Dermatol 2004; 43: 494-7.

35. Gurer MA, Erel A, Erbas D, Caglar K, Atahan C. The seroprevalence of Helicobacter pylori and nitric oxide in acne rosacea. Int J Dermatol 2002; 41: 768-70.

36. El-Khalawany $\mathrm{M}$, et al. Role of Helicobacter pylori in common rosacea subtypes: a genotypic comparative study of Egyptian patients. J Dermatol 2012; 39: 989-95.

37. Song EJ, Lee ES, Nam YD. Progress of analytical tools and techniques for human gut microbiome research. J Microbiol 2018; 56: 693-705. 
38. Cao Y, Fanning S, Proos S, Jordan K, Srikumar S. A review on the applications of next generation sequencing technologies as applied to food-related microbiome studies. Front Microbiol 2017; 8: 1829.

39. Grice EA. The skin microbiome: potential for novel diagnostic and therapeutic approaches to cutaneous disease. Semin Cutan Med Surg 2014; 33: 98-103.

40. Perez Perez GI, et al. Body site is a more determinant factor than human population diversity in the healthy skin microbiome. PloS one 2016; 11: e0151990.

41. Musthaq S, Mazuy A, Jakus J. The microbiome in dermatology. Clin Dermatol 2018; 36: 390-8.

42. Grice EA, et al. Topographical and temporal diversity of the human skin microbiome. Science 2009; 324: 1190-2.

43. Thompson KG, et al. Comparison of the skin microbiota in acne and rosacea, Exp Dermatol 2020. http://doi.org/10.1111/exd.14098. Online ahead of print. 32278334.

44. Zaidi AK, et al. Characterization of the facial microbiome in twins discordant for rosacea. Exp Dermatol 2018; 27: 295-8.

45. Rainer BM, et al. Characterization and analysis of the skin microbiota in rosacea: a case-control study. Am J Clin Dermatol 2020; 21 : 139-47.

46. Holmes AD, Spoendlin J, Chien AL, Baldwin H, Chang ALS. Evidence-based update on rosacea comorbidities and their common physiologic pathways. J Am Acad Dermatol 2018; 78: 156-66.

47. Wu CY, et al. Risk of inflammatory bowel disease in patients with rosacea: results from a nationwide cohort study in Taiwan. J Am Acad Dermatol 2017; 76: 911-17.

48. Egeberg A, Weinstock LB, Thyssen EP, Gislason GH, Thyssen JP. Rosacea and gastrointestinal disorders: a population-based cohort study. Br J Dermatol 2017; 176: 100-6.

49. Drago F, et al. The role of small intestinal bacterial overgrowth in rosacea: a 3-year follow-up. J Am Acad Dermatol 2016; 75 : e113-15.

50. Agnoletti AF, et al. Etiopathogenesis of rosacea: a prospective study with a three-year follow-up. G Ital Dermatol Venereol 2017; 152: 418-23.

51. Drago F, Ciccarese G, Parodi A. Effects of the treatment for small intestine bacterial overgrowth on rosacea. J Dermatol 2017; 44: e321.

52. Parodi A, et al. Small intestinal bacterial overgrowth in rosacea: clinical effectiveness of its eradication. Clin Gastroenterol Hepatol 2008; 6: 759-64.

53. Chen YJ, Lee WH, Ho HJ, Tseng CH, Wu CY. An altered fecal microbial profiling in rosacea patients compared to matched controls, J Formos Med Assoc 2020; 120: 256-64. http://doi.org/10. 1016/j.jfma.2020.04.034. 32446756.

54. Nam JH, et al. Rosacea and its association with enteral microbiota in Korean females. Exp Dermatol 2018; 27: 37-42.

55. Sener S, et al. Thiol/disulfide homeostasis as a marker of oxidative stress in rosacea: a controlled spectrophotometric study. Cutan Ocul Toxicol 2019; 38: 55-8.
56. Brescoll J, Daveluy S. A review of vitamin B12 in dermatology. Am J Clin Dermatol 2015; 16: 27-33.

57. McLaughlin RW, et al. Are there naturally occurring pleomorphic bacteria in the blood of healthy humans?. J Clin Microbiol 2002; 40: 4771-5.

58. Paisse S, et al. Comprehensive description of blood microbiome from healthy donors assessed by $16 \mathrm{~S}$ targeted metagenomic sequencing. Transfusion 2016; 56: 1138-47.

59. Mitchell AJ, et al. Pleomorphic structures in human blood are red blood cell-derived microparticles, not bacteria. PloS one 2016; 11: e0163582.

60. Qiu J, Zhou H, Jing Y, Dong C. Association between blood microbiome and type 2 diabetes mellitus: a nested case-control study. J Clin Lab Anal 2019; 33: e22842.

61. Olde Loohuis LM, et al. Transcriptome analysis in whole blood reveals increased microbial diversity in schizophrenia. Transl Psychiatry 2018; 8: 96.

62. Whittle E, Leonard MO, Harrison R, Gant TW, Tonge DP. Multimethod characterization of the human circulating microbiome. Front Microbiol 2018; 9: 3266.

63. Li Q, et al. Identification and characterization of blood and neutrophil-associated microbiomes in patients with severe acute pancreatitis using next-generation sequencing. Front Cell Infect Microbiol 2018; 8: 5.

64. Ring HC, et al. Moderate to severe hidradenitis suppurativa patients do not have an altered bacterial composition in peripheral blood compared to healthy controls. J Eur Acad Dermatol Venereol 2018; 32: $125-8$.

65. Markova ND. Eubiotic vs. dysbiotic human blood microbiota: the phenomenon of cell wall deficiency and disease-trigger potential of bacterial and fungal L-forms. Discov Med 2020; 29: 31-40.

66. Yun Y, et al. Characterization of the blood microbiota in Korean females with rosacea. Dermatology 2019; 235: 255-9.

67. Kumar A, et al. Taxonomic description and genome sequence of Rheinheimera mesophila sp. nov., isolated from an industrial waste site. Int J Syst Evol Microbiol 2015; 65: 3666-73.

68. Chen WM, Chen WT, Young CC, Sheu SY. Rheinheimera riviphila sp. nov., isolated from a freshwater stream. Arch Microbiol 2019; 201: 919-26.

69. Baek K, Jeon CO. Rheinheimera aestuari sp. nov., a marine bacterium isolated from coastal sediment. Int J Syst Evol Microbiol 2015; 65: 2640-5.

70. Ryu SH, et al. Rheinheimera soli sp. nov., a gammaproteobacterium isolated from soil in Korea. Int J Syst Evol Microbiol 2008; 58: 2271-4.

71. Becerra-Castro C, Macedo G, Silva AMT, Manaia CM, Nunes OC. Proteobacteria become predominant during regrowth after water disinfection. Sci Total Environ 2016; 573: 313-23.

72. Kuchly B, Aucher P, Violette J, Bourdiol MC. Rheinheimera bacteremia. Med Mal Infect 2020; 50: 377-8. 